\title{
Non-Constant Positive Steady States for a Predator-Prey Cross-Diffusion Model with Beddington-DeAngelis Functional Response
}

\author{
Lina Zhang and Shengmao Fu \\ Department of Mathematics, Northwest Normal University, Lanzhou 730070, China \\ Correspondence should be addressed to Shengmao Fu, fusm@nwnu.edu.cn
}

Received 13 October 2010; Accepted 30 January 2011

Academic Editor: Dumitru Motreanu

Copyright (c) 2011 L. Zhang and S. Fu. This is an open access article distributed under the Creative Commons Attribution License, which permits unrestricted use, distribution, and reproduction in any medium, provided the original work is properly cited.

This paper deals with a predator-prey model with Beddington-DeAngelis functional response under homogeneous Neumann boundary conditions. We mainly discuss the following three problems: (1) stability of the nonnegative constant steady states for the reaction-diffusion system; (2) the existence of Turing patterns; (3) the existence of stationary patterns created by crossdiffusion.

\section{Introduction}

Consider the following predator-prey system with diffusion:

$$
\begin{gathered}
u_{t}-d_{1} \Delta u=r_{1} u\left(1-\frac{u}{K}\right)-f v, \quad x \in \Omega, t>0, \\
v_{t}-d_{2} \Delta v=r_{2} v\left(1-\frac{v}{\delta u}\right), \quad x \in \Omega, t>0, \\
\partial_{v} u=\partial_{v} v=0, \quad x \in \partial \Omega, t>0, \\
u(x, 0)=u_{0}(x)>0, \quad v(x, 0)=v_{0}(x) \geq 0, \quad x \in \Omega,
\end{gathered}
$$

where $\Omega \subset \mathbb{R}^{N}$ is a bounded domain with smooth boundary $\partial \Omega$ and $v$ is the outward unit normal vector of the boundary $\partial \Omega$. In the system (1.1), $u(x, t)$ and $v(x, t)$ represent the densities of the species prey and predator, respectively, $u_{0}(x)$ and $v_{0}(x)$ are given smooth functions on $\bar{\Omega}$ which satisfy compatibility conditions. The constants $d_{1}, d_{2}$, called 
diffusion coefficients, are positive, $r_{1}$ and $r_{2}$ are the intrinsic growth rates of the prey and predator, $K$ denotes the carrying capacity of the prey, and $\delta u$ represents the carrying capacity of the predator, which is in proportion to the prey density. The function $f$ is a functional response function. The parameters $r_{1}, r_{2}, K$, and $\delta$ are all positive constants. The homogeneous Neumann boundary conditions indicate that the system is self-contained with zero population flux across the boundary. For more ecological backgrounds about this model, one can refer to [1-6].

In recent years there has been considerable interest in investigating the system (1.1) with the prey-dependent functional response (i.e., $f$ is only a function of $u$ ). In $[5,6], \mathrm{Du}$, Hsu and Wang investigated the global stability of the unique positive constant steady state and gained some important conclusions about pattern formation for (1.1) with Leslie-Gower functional response (i.e., $f=\beta u$ ). In [7, 8], Peng and Wang studied the long time behavior of time-dependent solutions and the global stability of the positive constant steady state for (1.1) with Holling-Tanner-type functional response (i.e., $f=\beta u /(m+u)$ ). They also established some results for the existence and nonexistence of non-constant positive steady states with respect to diffusion and cross-diffusion rates. In [9], Ko and Ryu investigated system (1.1) when $f$ satisfies a general hypothesis: $f(0)=0$, and there exists a positive constant $M$ such that $0<f_{u}(u) \leq M$ for all $u>0$. They studied the global stability of the positive constant steady state and derived various conditions for the existence and non-existence of non-constant positive steady states. When the function $f$ in the system (1.1) takes the form $f=\beta u /(u+m v)$ called ratio-dependent functional response, Peng, and Wang [10] studied the global stability of the unique positive constant steady state and gained several results for the non-existence of non-constant positive solutions.

It is known that the prey-dependent functional response means that the predation behavior of the predator is only determined by the prey, which contrasts with some realistic observations, such as the paradox of enrichment [11, 12]. The ratio-dependent functional response reflects the mutual interference between predator and prey, but it usually raises controversy because of the low-density problem [13]. In 1975, Beddington and DeAngelis $[14,15]$ proposed a function $f=\beta u /(1+m u+n v)$, commonly known as BeddingtonDeAngelis functional response. It has an extra term in the denominator which models mutual interference between predator and prey. In addition, it avoids the low-density problem.

In this paper, we study the system (1.1) with $f=\beta u /(1+m u+n v)$. Using the scaling

$$
\frac{r_{1}}{K} u \longrightarrow u, \quad \frac{r_{1}}{K \delta} v \longrightarrow v, \quad r_{1} \longrightarrow \lambda, \quad \frac{K \delta}{r_{1}} \beta \longrightarrow \beta, \quad \frac{K}{r_{1}} m \longrightarrow m, \quad \frac{K \delta}{r_{1}} n \longrightarrow n,
$$

and taking $r_{2}=1$ for simplicity of calculation, (1.1) becomes

$$
\begin{gathered}
u_{t}-d_{1} \Delta u=\lambda u-u^{2}-\frac{\beta u v}{1+m u+n v} \triangleq g_{1}(u, v), \quad x \in \Omega, t>0, \\
v_{t}-d_{2} \Delta v=v\left(1-\frac{v}{u}\right) \triangleq g_{2}(u, v), \quad x \in \Omega, t>0, \\
\partial_{v} u=\partial_{v} v=0, \quad x \in \partial \Omega, t>0, \\
u(x, 0)=u_{0}(x)>0, \quad v(x, 0)=v_{0}(x) \geq 0, \quad x \in \Omega .
\end{gathered}
$$


It is obvious that (1.3) has two nonnegative constant solutions: the semitrivial solution $(\lambda, 0)$ and the unique positive constant solution $\left(u_{*}, v_{*}\right)$, where

$$
u_{*}=\frac{\lambda(m+n)-1-\beta+\sqrt{[\lambda(m+n)-1-\beta]^{2}+4 \lambda(m+n)}}{2(m+n)}, \quad v_{*}=u_{*}
$$

In the system (1.3), the Beddington-DeAngelis functional response is used only in the prey equation, not the predator, and the predator equation contains a Leslie-Gower term $v /(\delta u)$ [16]. To our knowledge, there are few known results for (1.3) while there has been relatively good success for the predator-prey model with the full Beddington-DeAngelis functional responses. For example, Cantrell and Cosner [17] derived criteria for permanence and for predator extinction, and Chen and Wang [18] proved the nonexistence and existence of nonconstant positive steady states.

Taking into account the population fluxes of one species due to the presence of the other species, we consider the following cross-diffusion system:

$$
\begin{gathered}
u_{t}-d_{1} \Delta u=\lambda u-u^{2}-\frac{\beta u v}{1+m u+n v}, \quad x \in \Omega, t>0, \\
v_{t}-d_{2} \Delta\left(1+d_{3} u\right) v=v\left(1-\frac{v}{u}\right), \quad x \in \Omega, t>0, \\
\partial_{v} u=\partial_{v} v=0, \quad x \in \partial \Omega, t>0, \\
u(x, 0)=u_{0}(x)>0, \quad v(x, 0)=v_{0}(x) \geq 0 . \quad x \in \Omega,
\end{gathered}
$$

where $\Delta d_{2} d_{3} u v$ is a cross-diffusion term. If $d_{3}>0$, the movement of the predator is directed towards the lower concentration of the prey, which represents that the prey species congregate and form a huge group to protect themselves from the attack of the predator. It is clear that such an environment of prey-predator interaction often occurs in reality. For example, in [19-21], and so forth, with the similar biological interpretation, the authors also introduced the same cross-diffusion term as in (1.5) to the prey of various prey-predator models.

The main aim of this paper is to study the effects of the diffusion and crossdiffusion pressures on the existence of stationary patterns. We will demonstrate that the unique positive constant steady state $\left(u_{*}, v_{*}\right)$ for the reduced ODE system is locally asymptotically stable if $a_{11}<1$, where $a_{11}=1 / \beta\left\{m\left(\lambda-u_{*}\right)^{2}-\beta u_{*}\right\}$. But $\left(u_{*}, v_{*}\right)$ can lose its stability when it is regarded as a stationary solution of the corresponding reaction-diffusion system (see Theorem 2.5) and Turing patterns can be found as a result of diffusion (see Theorem 3.5). Moreover, after the cross-diffusion pressure is introduced, even though the unique positive constant steady state is asymptotically stable for the model without crossdiffusion, stationary patterns can also exist due to the emergence of cross-diffusion (see Theorem 4.4). The main conclusions of this paper continue to hold for any positive constant $r_{2}$. We also remark here that, there have been some works which are devoted to the studies of the role of diffusion and cross-diffusion in helping to create stationary patterns from the biological processes [22-25]. 
This paper is organized as follows. In Section 2, we study the long time behavior of (1.3). In Section 3, we investigate the existence of Turing patterns of (1.3) by using the LeraySchauder degree theory. In Section 4, we prove the existence of stationary patterns of (1.5). We end with a brief section on conclusions.

\section{The Long Time Behavior of Time-Dependent Solutions}

In this section, we discuss the global behavior of solutions for the system (1.3). By the standard theory of parabolic equations [26, 27], we can prove that the problem (1.3) has a unique classical global solution $(u, v)$, which satisfies $0<u(x, t) \leq \max \left\{\lambda, \sup _{\Omega} u_{0}\right\}$ and $0<v(x, t) \leq \max \left\{\lambda, \sup _{\Omega} u_{0}, \sup _{\Omega} v_{0}\right\}$ on $\bar{\Omega} \times[0,+\infty)$.

\subsection{Global Attractor and Permanence}

First, we show that $\Re_{0} \triangleq[0, \lambda] \times[0, \lambda]$ is a global attractor for $(1.3)$.

Theorem 2.1. Let $(u(x, t), v(x, t))$ be any non-negative solution of (1.3). Then,

$$
\lim _{t \rightarrow+\infty} \sup _{\bar{\Omega}} u(x, t) \leq \lambda, \quad \lim _{t \rightarrow+\infty} \sup _{\bar{\Omega}} v(x, t) \leq \lambda
$$

Proof. The first result of (2.1) follows easily from the comparison argument for parabolic problems. Then, there exists a constant $T \gg 0$ such that $u(x, t)<\lambda+\varepsilon$ on $\bar{\Omega} \times[T,+\infty)$ for an arbitrary constant $\varepsilon>0$, and thus,

$$
v_{t}-d_{2} \Delta v \leq v\left(1-\frac{v}{\lambda+\varepsilon}\right), \quad(x, t) \in \Omega \times[T,+\infty) .
$$

Let $v(t)$ be the unique positive solution of

$$
\begin{gathered}
\frac{d w}{d t}=w\left(1-\frac{w}{\lambda+\varepsilon}\right), \quad t \in[T,+\infty), \\
w(T)=\max _{\bar{\Omega}} v(x, T) \geq 0 .
\end{gathered}
$$

The comparison argument yields

$$
\lim _{t \rightarrow+\infty} \sup _{\bar{\Omega}} v(x, t) \leq \lim _{t \rightarrow+\infty} v(t)=\lambda+\varepsilon
$$

which implies the second assertion of (2.1) by the continuity as $\varepsilon \rightarrow 0$.

Theorem 2.2. Assume that $\beta<n \lambda+1$, then the positive solution $(u(x, t), v(x, t))$ of $(1.3)$ satisfies

$$
\lim _{t \rightarrow+\infty} \inf _{\bar{\Omega}} u(x, t) \geq K, \quad \lim _{t \rightarrow+\infty} \inf _{\bar{\Omega}} v(x, t) \geq K,
$$


where

$$
K \triangleq \frac{1}{2 m}\left\{(m-n) \lambda-1+\sqrt{[(m-n) \lambda-1]^{2}+4 m \lambda(1+n \lambda-\beta)}\right\}
$$

Proof. Since $\beta<n \lambda+1$, there exists a sufficiently small constant $\varepsilon_{1}>0$ such that $\lambda+(n \lambda-\beta)(\lambda+$ $\left.\varepsilon_{1}\right)>0$. In view of Theorem 2.1, there exists a $T \gg 0$ such that $v(x, t)<\lambda+\varepsilon_{1}$ in $\bar{\Omega} \times[T,+\infty)$. Thus we have

$$
u_{t}-d_{1} \Delta u \geq \frac{-m u^{2}+\left(m \lambda-n \lambda-n \varepsilon_{1}-1\right) u+\lambda+(n \lambda-\beta)\left(\lambda+\varepsilon_{1}\right)}{1+m u+n\left(\lambda+\varepsilon_{1}\right)} u
$$

for $(x, t) \in \bar{\Omega} \times[T,+\infty)$. Let $u(t)$ be the unique positive solution of

$$
\begin{gathered}
\frac{d w}{d t}=\frac{-m w^{2}+\left(m \lambda-n \lambda-n \varepsilon_{1}-1\right) w+\lambda+(n \lambda-\beta)\left(\lambda+\varepsilon_{1}\right)}{1+m w+n\left(\lambda+\varepsilon_{1}\right)} w, \quad t \in[T,+\infty), \\
w(T)=\min _{\bar{\Omega}} u(x, T)>0 .
\end{gathered}
$$

Then, $\lim _{t \rightarrow+\infty} \inf _{\bar{\Omega}} u(x, t) \geq \lim _{t \rightarrow+\infty} u(t)$, where

$$
\lim _{t \rightarrow+\infty} u(t)=\frac{1}{2 m}\left\{(m-n) \lambda-1-n \varepsilon_{1}+\sqrt{\left[(m-n) \lambda-n \varepsilon_{1}-1\right]^{2}+4 m\left[\lambda+(n \lambda-\beta)\left(\lambda+\varepsilon_{1}\right)\right]}\right\}
$$

By continuity as $\varepsilon_{1} \rightarrow 0$, we have $\lim _{t \rightarrow+\infty} \inf _{\bar{\Omega}} u(x, t) \geq K$. Similarly, we can prove the second result of (2.5).

From Theorems 2.1 and 2.2, we see that the system (1.3) is permanent if $\beta<n \lambda+1$.

\subsection{Local Stability of Nonnegative Equilibria}

Now, we consider the stability of non-negative equilibria.

Lemma 2.3. The semi-trivial solution $(\lambda, 0)$ of $(1.3)$ is unconditionally unstable.

Proof. The linearization matrix of $(1.3)$ at $(\lambda, 0)$ is

$$
J_{1}=\left(\begin{array}{cc}
-\lambda & -\frac{\beta \lambda}{1+m \lambda} \\
0 & 1
\end{array}\right)
$$

It is easy to see that 1 is an eigenvalue of $J_{1}$, thus $(\lambda, 0)$ is unconditionally unstable. 
Now, we discuss the Turing instability of $\left(u_{*}, v_{*}\right)$. Recall that a constant solution is Turing unstable if it is stable in the absence of diffusion, and it becomes unstable when diffusion is present [28]. More precisely, this requires the following two conditions.

(i) It is stable as an equilibrium of the system of ordinary differential equations

$$
\frac{d u}{d t}=g_{1}(u, v), \quad \frac{d v}{d t}=g_{2}(u, v),
$$

where $g_{1}(u, v)$ and $g_{2}(u, v)$ are given in (1.3).

(ii) It is unstable as a steady state of the reaction-diffusion system (1.3).

Theorem 2.4. If $a_{11}<1$, then the unique positive equilibrium $\left(u_{*}, v_{*}\right)$ of $(2.11)$ is locally asymptotically stable. If $a_{11}>1$, then $\left(u_{*}, v_{*}\right)$ is unstable, where $a_{11}=1 / \beta\left[m\left(\lambda-u_{*}\right)^{2}-\beta u_{*}\right]$.

Proof. The linearization matrix of $(2.11)$ at $\left(u_{*}, v_{*}\right)$ is

$$
J_{2}=\left(\begin{array}{ll}
a_{11} & a_{12} \\
a_{21} & a_{22}
\end{array}\right)
$$

where

$$
a_{11}=\frac{1}{\beta}\left[m\left(\lambda-u_{*}\right)^{2}-\beta u_{*}\right], \quad a_{12}=-\frac{\left(\lambda-u_{*}\right)\left(1+m u_{*}\right)}{1+(m+n) u_{*}}, \quad a_{21}=1, \quad a_{22}=-1 .
$$

A simple calculation shows

$$
\operatorname{det} J_{2}=-a_{11}-a_{12}=\frac{(m+n) u_{*}^{2}+\lambda}{1+(m+n) u_{*}}, \quad \text { trace } J_{2}=a_{11}-1
$$

Clearly, det $J_{2}>0$. If $a_{11}<1$, then trace $J_{2}<0$. Hence, all eigenvalues of $J_{2}$ have negative real parts and $\left(u_{*}, v_{*}\right)$ is locally asymptotically stable. If $a_{11}>1$, then trace $J_{2}>0$, which implies that $J_{2}$ has two eigenvalues with positive real parts and $\left(u_{*}, v_{*}\right)$ is unstable.

Similarly as in $[23,29]$, let $0=\mu_{1}<\mu_{2}<\mu_{3}<\mu_{4} \ldots$ be the eigenvalues of the operator $-\Delta$ on $\Omega$ with the homogeneous Neumann boundary condition, and let $E\left(\mu_{i}\right)$ be the eigenspace corresponding to $\mu_{i}$ in $H^{1}(\Omega)$. Let $\left\{\phi_{i j}: j=1,2, \ldots, \operatorname{dim} E\left(\mu_{i}\right)\right\}$ be the orthonormal basis of $E\left(\mu_{i}\right), \mathbf{X}=\left[H^{1}(\Omega)\right]^{2}, \mathbf{X}_{i j}=\left\{\mathbf{c} \phi_{i j}: \mathbf{c} \in \mathbb{R}^{2}\right\}$. Then,

$$
\mathbf{X}=\bigoplus_{i=1}^{+\infty} \mathbf{X}_{i}, \quad \mathbf{X}_{i}=\bigoplus_{j=1}^{\operatorname{dim} E\left(\mu_{i}\right)} \mathbf{X}_{i j}
$$

Define $i_{0}$ as the largest positive integer such that $d_{1} \mu_{i}<a_{11}$ for $i \leq i_{0}$. Clearly, if

$$
d_{1} \mu_{2}<a_{11}
$$


then $2 \leq i_{0}<+\infty$. In this case, denote

$$
\tilde{d}_{2} \triangleq \min _{2 \leq i \leq i_{0}} d_{2}^{(i)}, \quad d_{2}^{(i)} \triangleq \frac{d_{1} \mu_{i}+\operatorname{det} J_{2}}{\mu_{i}\left(a_{11}-d_{1} \mu_{i}\right)}
$$

The local stability of $\left(u_{*}, v_{*}\right)$ for (1.3) can be summarized as follows.

Theorem 2.5. (i) Assume that $a_{11}>1$, then $\left(u_{*}, v_{*}\right)$ is unstable.

(ii) Assume that $a_{11}<1$. Then $\left(u_{*}, v_{*}\right)$ is locally asymptotically stable if $a_{11} \leq d_{1} \mu_{2} ;\left(u_{*}, v_{*}\right)$ is locally asymptotically stable if $a_{11}>d_{1} \mu_{2}$ and $d_{2}<\tilde{d}_{2} ;\left(u_{*}, v_{*}\right)$ is unstable if $a_{11}>d_{1} \mu_{2}$ and $d_{2}>\tilde{d}_{2}$.

Proof. Consider the following linearization operator of $(1.3)$ at $\left(u_{*}, v_{*}\right)$ :

$$
L=\left(\begin{array}{cc}
d_{1} \Delta+a_{11} & a_{12} \\
a_{21} & d_{2} \Delta+a_{22}
\end{array}\right)
$$

where $a_{11}, a_{12}, a_{21}$, and $a_{22}$ are given in (2.13). Suppose $(\phi(x), \psi(x))^{T}$ is an eigenfunction of $L$ corresponding to an eigenvalue $\tilde{\mu}$, then

$$
\left(d_{1} \Delta \phi+\left(a_{11}-\tilde{\mu}\right) \phi+a_{12} \psi, d_{2} \Delta \psi+a_{21} \phi+\left(a_{22}-\tilde{\mu}\right) \psi\right)^{T}=(0,0)^{T} .
$$

Setting

$$
\phi=\sum_{1 \leq i<+\infty,} a_{1 \leq j \leq \operatorname{dim} E\left(\mu_{i}\right)} \phi_{i j}, \quad \psi=\sum_{1 \leq i<+\infty,} \sum_{1 \leq j \leq \operatorname{dim} E\left(\mu_{i}\right)} b_{i j} \phi_{i j}
$$

we can find that

$$
\sum_{1 \leq i<+\infty,} L_{i \leq j \leq \operatorname{dim} E\left(\mu_{i}\right)}\left(\begin{array}{l}
a_{i j} \\
b_{i j}
\end{array}\right) \phi_{i j}=0, \quad \text { where } L_{i}=\left(\begin{array}{cc}
a_{11}-d_{1} \mu_{i}-\tilde{\mu} & a_{12} \\
a_{21} & a_{22}-d_{2} \mu_{i}-\tilde{\mu}
\end{array}\right) .
$$

It follows that $\tilde{\mu}$ is an eigenvalue of $L$ if and only if the determinant of the matrix $L_{i}$ is zero for some $i \geq 1$, that is,

$$
\tilde{\mu}^{2}+P_{i} \tilde{\mu}+Q_{i}=0
$$

where

$$
P_{i}=\left(d_{1}+d_{2}\right) \mu_{i}-\operatorname{trace} J_{2}, \quad Q_{i}=-d_{2} \mu_{i}\left(a_{11}-d_{1} \mu_{i}\right)+d_{1} \mu_{i}+\operatorname{det} J_{2}
$$

Clearly, $Q_{1}>0$ since $\mu_{1}=0$. If $a_{11}>1$, then trace $J_{2}>0$ and $P_{1}<0$. Hence, $L$ has two eigenvalues with positive real parts and the steady state $\left(u_{*}, v_{*}\right)$ is unstable. 
Note that $P_{i}>0$ for all $i \geq 1$ if $a_{11}<1$, and $Q_{i}>0$ for all $i \geq 1$ if $a_{11} \leq d_{1} \mu_{2}$. This implies that $\operatorname{Re} \tilde{\mu}<0$ for all eigenvalue $\tilde{\mu}$, and so the steady state $\left(u_{*}, v_{*}\right)$ is locally asymptotically stable.

Assume that $a_{11}>d_{1} \mu_{2}$. If $d_{2}<\tilde{d}_{2}$, then $d_{1} \mu_{i}<a_{11}$ and $d_{2}<d_{2}^{(i)}$ for $i \in\left[2, i_{0}\right]$. It follows that $Q_{i}>0$ for all $i \in\left[2, i_{0}\right]$. Furthermore, if $i>i_{0}$, then $d_{1} \mu_{i} \geq a_{11}$ and $Q_{i}>0$. The conclusion leads to the locally asymptotically stability of $\left(u_{*}, v_{*}\right)$ again. If $d_{2}>\tilde{d}_{2}$, then we may assume that the minimum in (2.17) is attained by $k \in\left[2, i_{0}\right]$. Thus, $d_{1} \mu_{k}<a_{11}$ and $d_{2}>d_{2}^{(k)}$, so we have $Q_{k}<0$. This implies that $\left(u_{*}, v_{*}\right)$ is unstable.

Remark 2.6. From Theorems 2.4 and 2.5, we can conclude that $\left(u_{*}, v_{*}\right)$ is Turing unstable if $d_{1} \mu_{2}<a_{11}<1$ and $d_{2}>\tilde{d}_{2}$.

\subsection{Global Stability of $\left(u_{*}, v_{*}\right)$}

The following three theorems are the global stability results of the positive constant solution $\left(u_{*}, v_{*}\right)$. In the sense of biology, our conclusion of the global stability of $\left(u_{*}, v_{*}\right)$ implies that, in some ranges of the parameters $\lambda, \beta, m$, and $n$, both the prey and the predator will be spatially homogeneously distributed as time converges to infinity, no matter how quickly or slowly they diffuse.

Theorem 2.7. Assume that $\beta<n \lambda+1$ and

$$
\beta\left\{\frac{\lambda+u_{*}}{K+u_{*}}\left(1+m u_{*}\right)-\frac{1+m K+n K}{1+m \lambda+n \lambda}\right\}<\left(1+m u_{*}+n v_{*}\right)(1+m K+n K)
$$

Then $\left(u_{*}, v_{*}\right)$ attracts all positive solutions of (1.3).

Proof. Define the Lyapunov function

$$
E_{1}(t)=\int_{\Omega}\left(u-2 u_{*}+\frac{u_{*}^{2}}{u}\right) d x+\delta_{1} \int_{\Omega}\left(v-v_{*}-v_{*} \ln \frac{v}{v_{*}}\right) d x
$$

where

$$
\delta_{1}=\left(K+u_{*}\right)\left\{1+\frac{\beta}{\left(1+m u_{*}+n v_{*}\right)(1+m \lambda+n \lambda)}\right\}
$$

$(u, v)$ is a positive solution of (1.3). Then $E_{1}(t) \geq 0$ for all $t \geq 0$. The straightforward computations give that

$$
\begin{aligned}
\frac{d E_{1}}{d t} & =\int_{\Omega} \frac{u^{2}-u_{*}{ }^{2}}{u^{2}} u_{t} d x+\delta_{1} \int_{\Omega} \frac{v-v_{*}}{v} v_{t} d x \\
& =\int_{\Omega} D_{1} d x+\int_{\Omega} \frac{1}{u}\left\{A_{1}\left(u-u_{*}\right)^{2}+B_{1}\left(u-u_{*}\right)\left(v-v_{*}\right)+C_{1}\left(v-v_{*}\right)^{2}\right\} d x,
\end{aligned}
$$


where

$$
\begin{gathered}
D_{1}=-\left\{d_{1} \frac{2 u_{*}^{2}}{u^{3}}|\nabla u|^{2}+\delta_{1} d_{2} \frac{v_{*}}{v^{2}}|\nabla v|^{2}\right\} \leq 0, \\
A_{1}=\left(u+u_{*}\right)\left\{-1+\frac{\beta m v_{*}}{\left(1+m u_{*}+n v_{*}\right)(1+m u+n v)}\right\}, \\
B_{1}=\delta_{1}-\frac{\beta\left(u+u_{*}\right)\left(1+m u_{*}\right)}{\left(1+m u_{*}+n v_{*}\right)(1+m u+n v)}, \quad C_{1}=-\delta_{1} .
\end{gathered}
$$

From Theorems 2.1 and 2.2, there exists a $t_{0} \gg 0$ such that $K-\varepsilon<u(x, t), v(x, t)<\lambda+\varepsilon$ in $\bar{\Omega} \times\left[t_{0},+\infty\right)$ for an arbitrary and small enough constant $\varepsilon>0$. By continuity as $\varepsilon \rightarrow 0,(2.24)$ implies that

$$
\begin{aligned}
B_{1}= & \frac{K+u_{*}}{\left(1+m u_{*}+n v_{*}\right)(1+m K+n K)} \\
& \times\left\{\left(1+m u_{*}+n v_{*}\right)(1+m K+n K)\right. \\
& \left.\quad-\beta\left(\frac{\left(u+u_{*}\right)\left(1+m u_{*}\right)(1+m K+n K)}{\left(K+u_{*}\right)(1+m u+n v)}-\frac{1+m K+n K}{1+m \lambda+n \lambda}\right)\right\} \geq 0
\end{aligned}
$$

in $\bar{\Omega} \times\left[t_{0},+\infty\right)$. Applying the Young inequality to (2.27), we have

$$
\begin{aligned}
\frac{d E_{1}}{d t} \leq & \int_{\Omega} D_{1} d x+\int_{\Omega} \frac{1}{u}\left(A_{1}+B_{1}\right)\left(u-u_{*}\right)^{2} d x+\int_{\Omega} \frac{1}{u}\left(\frac{B_{1}}{4}+C_{1}\right)\left(v-v_{*}\right)^{2} d x \\
= & \int_{\Omega} D_{1} d x+\int_{\Omega} \frac{1}{u}\left\{\delta_{1}-\left(u+u_{*}\right)\left(1+\frac{\beta}{\left(1+m u_{*}+n v_{*}\right)(1+m u+n v)}\right)\right\}\left(u-u_{*}\right)^{2} d x \\
& +\int_{\Omega} \frac{1}{u}\left\{-\frac{3}{4} \delta_{1}-\frac{\beta\left(u+u_{*}\right)\left(1+m u_{*}\right)}{4\left(1+m u_{*}+n v_{*}\right)(1+m u+n v)}\right\}\left(v-v_{*}\right)^{2} d x \\
\leq & 0
\end{aligned}
$$

in $\bar{\Omega} \times\left[t_{0},+\infty\right)$. Similarly as in [24,30], the standard argument concludes $(u(x, t), v(x, t)) \rightarrow$ $\left(u_{*}, v_{*}\right)$ in $\left[L^{\infty}(\Omega)\right]^{2}$, which thereby shows that $\left(u_{*}, v_{*}\right)$ attracts all positive solutions of $(1.3)$ under our hypotheses. Thus, the proof is complete. 
Theorem 2.8. Assume that $\beta<n \lambda+1$,

$$
\begin{gathered}
\beta\left(1+m u_{*}-\frac{1+m K+n K}{1+m \lambda+n \lambda}\right)<\left(1+m u_{*}+n v_{*}\right)(1+m K+n K), \\
\beta<\frac{(\lambda m+\lambda n+2)(m+n)}{2} .
\end{gathered}
$$

Then, $\left(u_{*}, v_{*}\right)$ attracts all positive solutions of (1.3).

Proof. Define the Lyapunov function

$$
E_{2}(t)=\int_{\Omega}\left\{\frac{u_{*}-u}{u}+\ln \frac{u}{u_{*}}\right\} d x+\delta_{2} \int_{\Omega}\left\{v-v_{*}-v_{*} \ln \frac{v}{v_{*}}\right\} d x
$$

where $\delta_{2}=1+\left(\beta /\left(1+m u_{*}+n v_{*}\right)(1+m \lambda+n \lambda),(u, v)\right)$ is a positive solution of (1.3). Then

$$
\frac{d E_{2}}{d t}=\int_{\Omega} D_{2} d x+\int_{\Omega} \frac{1}{u}\left\{A_{2}\left(u-u_{*}\right)^{2}+B_{2}\left(u-u_{*}\right)\left(v-v_{*}\right)+C_{2}\left(v-v_{*}\right)^{2}\right\} d x,
$$

where

$$
\begin{gathered}
D_{2}=-\left\{d_{1} \frac{2 u_{*}-u}{u^{3}}|\nabla u|^{2}+\delta_{2} d_{2} \frac{v_{*}}{v^{2}}|\nabla v|^{2}\right\}, \\
A_{2}=-1+\frac{\beta m v_{*}}{\left(1+m u_{*}+n v_{*}\right)(1+m u+n v)^{\prime}}, \\
B_{2}=\delta_{2}-\frac{\beta\left(1+m u_{*}\right)}{\left(1+m u_{*}+n v_{*}\right)(1+m u+n v)}, \quad C_{2}=-\delta_{2} .
\end{gathered}
$$

From Theorems 2.1 and 2.2, there exists a $t_{0} \gg 0$ such that $K-\varepsilon<u(x, t), v(x, t)<\lambda+\varepsilon$ in $\bar{\Omega} \times\left[t_{0},+\infty\right)$ for an arbitrary and small enough constant $\varepsilon>0$. Thus (2.31) implies that

$$
\begin{aligned}
B_{2}= & \frac{1}{\left(1+m u_{*}+n v_{*}\right)(1+m K+n K)} \\
& \times\left\{\left(1+m u_{*}+n v_{*}\right)(1+m \mathrm{~K}+n K)\right. \\
& \left.\quad-\beta\left(\frac{\left(1+m u_{*}\right)(1+m K+n K)}{(1+m u+n v)}-\frac{1+m K+n K}{1+m \lambda+n \lambda}\right)\right\} \geq 0
\end{aligned}
$$


in $\bar{\Omega} \times\left[t_{0},+\infty\right)$. On the other hand, (2.32) guarantees that $2 u_{*}-u>0$ in $\bar{\Omega} \times\left[t_{0},+\infty\right)$. Applying the Young inequality to (2.34), we have

$$
\begin{aligned}
\frac{d E_{2}}{d t} \leq & \int_{\Omega} D_{2} d x+\int_{\Omega} \frac{1}{u}\left(A_{2}+B_{2}\right)\left(u-u_{*}\right)^{2} d x+\int_{\Omega} \frac{1}{u}\left(\frac{B_{2}}{4}+C_{2}\right)\left(v-v_{*}\right)^{2} d x \\
= & \int_{\Omega} D_{2} d x+\int_{\Omega} \frac{1}{u}\left\{\delta_{2}-\left(1+\frac{\beta}{\left(1+m u_{*}+n v_{*}\right)(1+m u+n v)}\right)\right\}\left(u-u_{*}\right)^{2} d x \\
& +\int_{\Omega} \frac{1}{u}\left\{-\frac{3}{4} \delta_{2}-\frac{\beta\left(1+m u_{*}\right)}{4\left(1+m u_{*}+n v_{*}\right)(1+m u+n v)}\right\}\left(v-v_{*}\right)^{2} d x \\
\leq & 0
\end{aligned}
$$

in $\bar{\Omega} \times\left[t_{0},+\infty\right)$. Consequently, our analysis confirms that Theorem 2.8 holds.

Remark 2.9. If we choose the common Lyapunov function

$$
E_{3}(t)=\int_{\Omega}\left\{u-u_{*}-u_{*} \ln \frac{u}{u_{*}}\right\} d x+\delta_{3} \int_{\Omega}\left\{v-v_{*}-v_{*} \ln \frac{v}{v_{*}}\right\} d x
$$

where $\delta_{3}=K\left\{1+\left(\beta /\left(1+m u_{*}+n v_{*}\right)(1+m \lambda+n \lambda)\right)\right\}$, we can also derive the global stability of $\left(u_{*}, v_{*}\right)$ for (1.3) under a stronger condition than (2.24). Thus, the Lyapunov function defined by (2.25) is better than (2.38) in discussing the global stability of $\left(u_{*}, v_{*}\right)$ for (1.3).

Remark 2.10. If we choose $m=1$, then (2.32) holds since $\beta<\lambda n+1$. It is not hard to verify that the condition (2.31) in Theorem 2.8 contains the condition (2.24) in Theorem 2.7. However, if we choose $m$ and $n$ to be sufficiently small, then $u_{*}=v_{*} \rightarrow \lambda /(1+\beta)$ and $K \rightarrow \lambda(1-\beta)$. We can see that the range of parameters satisfying (2.24) is wider than that satisfying (2.32). This means that we can derive various conditions for the global stability of $\left(u_{*}, v_{*}\right)$ by choosing different Lyapunov functions.

\section{Stationary Patterns for the PDE System without Cross-Diffusion}

In this section, we discuss the corresponding steady-state problem of (1.3):

$$
\begin{gathered}
-d_{1} \Delta u=\lambda u-u^{2}-\frac{\beta u v}{1+m u+n v}=g_{1}(u, v) \quad \text { in } \Omega, \\
-d_{2} \Delta v=v\left(1-\frac{v}{u}\right)=g_{2}(u, v) \quad \text { in } \Omega, \\
\partial_{v} u=\partial_{v} v=0 \quad \text { on } \partial \Omega .
\end{gathered}
$$

The existence and non-existence of the non-constant positive solutions of (3.1) will be given.

In the following, the generic constants $C_{1}, C_{2}, C_{*}, \underline{C}, \bar{C}$, and so forth, will depend on the domain $\Omega$ and the dimension $N$. However, as $\Omega$ and the dimension $N$ are fixed, we will 
not mention the dependence explicitly. Also, for convenience, we shall write $\Lambda$ instead of the collective constants $(\lambda, \beta, m, n)$.

\subsection{A Priori Upper and Lower Bounds}

The main purpose of this subsection is to give a priori upper and lower bounds for the positive solutions to (3.1). To this aim, we first cite two known results.

Lemma 3.1 (maximum principle [25]). Let $g \in C\left(\Omega \times \mathbb{R}^{1}\right)$ and $b_{j} \in C(\bar{\Omega}), j=1,2, \ldots, N$.

(i) If $w \in C^{2}(\Omega) \cap C^{1}(\bar{\Omega})$ satisfies

$$
\begin{gathered}
\Delta w(x)+\sum_{j=1}^{N} b_{j}(x) w_{x_{j}}+g(x, w(x)) \geq 0 \quad \text { in } \Omega \\
\frac{\partial w}{\partial v} \leq 0 \quad \text { on } \partial \Omega
\end{gathered}
$$

and $w\left(x_{0}\right)=\max _{\bar{\Omega}} w(x)$, then $g\left(x_{0}, w\left(x_{0}\right)\right) \geq 0$.

(ii) If $w \in C^{2}(\Omega) \cap C^{1}(\bar{\Omega})$ satisfies

$$
\begin{gathered}
\Delta w(x)+\sum_{j=1}^{N} b_{j}(x) w_{x_{j}}+g(x, w(x)) \leq 0 \quad \text { in } \Omega \\
\frac{\partial w}{\partial v} \geq 0 \quad \text { on } \partial \Omega
\end{gathered}
$$

and $w\left(x_{0}\right)=\min _{\bar{\Omega}} w(x)$, then $g\left(x_{0}, w\left(x_{0}\right)\right) \leq 0$.

Lemma 3.2 (Harnack, inequality [31]). Let $w \in C^{2}(\Omega) \cap C^{1}(\bar{\Omega})$ be a positive solution to $\Delta w(x)+$ $c(x) w(x)=0$, where $c \in C(\bar{\Omega})$, satisfying the homogeneous Neumann boundary condition. Then there exists a positive constant $C_{*}$ which depends only on $\|c\|_{\infty}$ such that

$$
\max _{\bar{\Omega}} w \leq C_{*} \min _{\bar{\Omega}} w
$$

The results of upper and lower bounds can be stated as follows.

Theorem 3.3. For any positive number $d$, there exists a positive constant $\underline{C}(\Lambda, d)$ such that every positive solution $(u, v)$ of (3.1) satisfies $\underline{C}<u(x), v(x)<\lambda$ if $d_{1} \geq d$. 
Proof. Let $u\left(x_{1}\right)=\max _{\bar{\Omega}} u(x), v\left(x_{2}\right)=\max _{\bar{\Omega}} v(x), u\left(y_{1}\right)=\min _{\bar{\Omega}} u(x), v\left(y_{2}\right)=\min _{\bar{\Omega}} v(x)$. Application of Lemma 3.1 yields that

$$
\begin{gathered}
\lambda-u\left(x_{1}\right)-\frac{\beta v\left(x_{1}\right)}{1+m u\left(x_{1}\right)+n v\left(x_{1}\right)} \geq 0 \\
\lambda-u\left(y_{1}\right)-\frac{\beta v\left(y_{1}\right)}{1+m u\left(y_{1}\right)+n v\left(y_{1}\right)} \leq 0 \\
1-\frac{v\left(x_{2}\right)}{u\left(x_{2}\right)} \geq 0, \quad 1-\frac{v\left(y_{2}\right)}{u\left(y_{2}\right)} \leq 0 .
\end{gathered}
$$

Clearly, $u\left(x_{1}\right)<\lambda$ and $v\left(x_{2}\right) \leq u\left(x_{2}\right) \leq u\left(x_{1}\right)<\lambda$. Moreover, we have

$$
\begin{gathered}
v\left(y_{1}\right) \leq v\left(x_{2}\right) \leq u\left(x_{2}\right) \leq u\left(x_{1}\right), \\
v\left(y_{1}\right) \geq v\left(y_{2}\right) \geq u\left(y_{2}\right) \geq u\left(y_{1}\right) .
\end{gathered}
$$

By (3.5), we obtain

$$
m\left(u\left(y_{1}\right)\right)^{2}+\left[1+n v\left(y_{1}\right)-\lambda m\right] u\left(y_{1}\right)+(\beta-\lambda n) v\left(y_{1}\right)-\lambda \geq 0
$$

Noting that $u\left(y_{1}\right) \leq v\left(y_{1}\right) \leq u\left(x_{1}\right)$ from (3.6) and (3.7), (3.8) implies that $\max _{\bar{\Omega}} u(x)=u\left(x_{1}\right)>$ $C_{1}$ for some positive constant $C_{1}=C_{1}(\Lambda)$.

Let $c(x) \triangleq d_{1}^{-1}\left(\lambda-u-\left(\beta v /(1+m u+n v)\right.\right.$. Then, $\|c(x)\|_{\infty} \leq(2+\beta) \lambda / d$. The Harnack inequality shows that there exists a positive constant $C_{*}=C_{*}(\lambda, \beta, d)$ such that

$$
\max _{\bar{\Omega}} u(x) \leq C_{*} \min _{\bar{\Omega}} u(x)
$$

Combining (3.9) with $\max _{\bar{\Omega}} u(x)>C_{1}$, we find that $\min _{\bar{\Omega}} u(x)>C_{1}$ for some positive constant $\underline{C}=\underline{C}(\Lambda, d)$. It follows from (3.7) that $\min _{\bar{\Omega}} v(x)=v\left(y_{2}\right) \geq u\left(y_{1}\right)>\underline{C}$. The proof is completed.

\subsection{Non-Existence of Non-Constant Positive Steady States}

In the following theorem we will discuss the non-constant positive solutions to (3.1) when the diffusion coefficient $d_{1}$ varies while the other parameters $d_{2}, \lambda, \beta, m$, and $n$ are fixed.

Theorem 3.4. For any positive number $d$, there exists a positive constant $D=D(\Lambda, d)>d$ such that (3.1) has no non-constant positive solution if $d_{1}>D$.

Proof. For any $\varphi \in L^{1}(\Omega)$, let

$$
\bar{\varphi}=\frac{1}{|\Omega|} \int_{\Omega} \varphi d x
$$


Assume that $(u, v)$ is a positive solution of (3.1), multiplying the two equations of (3.1) by $(u-\bar{u}) / u$ and $(v-\bar{v}) / v$, respectively, and then integrating over $\Omega$ by parts, we have

$$
\begin{aligned}
\int_{\Omega}\left\{\frac{d_{1} \bar{u}}{u^{2}}|\nabla u|^{2}+\frac{d_{2} \bar{v}}{v^{2}}|\nabla v|^{2}\right\} d x= & \int_{\Omega} g_{1}(u, v) \frac{u-\bar{u}}{u} d x+\int_{\Omega} g_{2}(u, v) \frac{v-\bar{v}}{v} d x \\
= & \int_{\Omega}\left\{-1+\frac{\beta m \bar{v}}{(1+m \bar{u}+n \bar{v})(1+m u+n v)}\right\}(u-\bar{u})^{2} d x \\
& +\int_{\Omega}\left\{-\frac{\beta(1+m \bar{u})}{(1+m \bar{u}+n \bar{v})(1+m u+n v)}+\frac{\bar{v}}{u \bar{u}}\right\}(u-\bar{u})(v-\bar{v}) d x \\
& +\int_{\Omega}\left(-\frac{1}{u}\right)(v-\bar{v})^{2} d x .
\end{aligned}
$$

From Theorem 3.3 and Young's inequality, we obtain

$$
\int_{\Omega}\left\{d_{1}|\nabla u|^{2}+d_{2}|\nabla v|^{2}\right\} d x \leq C_{2}\left(-1+\frac{\beta m}{n}+C_{3}\right) \int_{\Omega}(u-\bar{u})^{2} d x+C_{2} \int_{\Omega}\left(\varepsilon-\frac{1}{u}\right)(v-\bar{v})^{2} d x
$$

for some positive constants $C_{2}=C_{2}(\Lambda, d), C_{3}=C_{3}(\Lambda, d, \varepsilon)$, where $\varepsilon$ is the arbitrary small positive constant arising from Young's inequality. By Theorem 3.3, we can choose $\varepsilon \in(0,1 / \lambda)$. Then applying the Poincaré inequality to (3.12) we obtain

$$
\mu_{2} \int_{\Omega}\left\{d_{1}(u-\bar{u})^{2}+d_{2}(v-\bar{v})^{2}\right\} d x \leq C_{4} \int_{\Omega}(u-\bar{u})^{2} d x+C_{2} \int_{\Omega}\left(\varepsilon-\frac{1}{u}\right)(v-\bar{v})^{2} d x
$$

which implies that $u=\bar{u}=$ constant and $v=\bar{v}=$ constant if $d_{1}>D=\max \left\{C_{4} / \mu_{2}, d\right\}$.

\subsection{Existence of Non-Constant Positive Steady States}

Throughout this subsection, we always assume that $a_{11}>0$. First, we study the linearization of (3.1) at $\left(u_{*}, v_{*}\right)$. Let

$$
\mathbf{Y}=\left\{(u, v):(u, v) \in\left[C^{1}(\bar{\Omega})\right]^{2}, \partial_{v} u=\partial_{v} v=0 \text { on } \partial \Omega\right\}
$$

For the sake of convenience, we define a compact operator $\mathcal{F}: \mathbf{Y} \rightarrow \mathbf{Y}$ by

$$
\mathcal{F}(\mathbf{e}) \triangleq\left(\begin{array}{c}
\left(a_{11}-d_{1} \Delta\right)^{-1}\left(g_{1}(u, v)+a_{11} u\right) \\
\left(-a_{22}-d_{2} \Delta\right)^{-1}\left(g_{2}(u, v)-a_{22} v\right)
\end{array}\right)
$$


where $\mathbf{e}=(u(x), v(x))^{T},\left(a_{11}-d_{1} \Delta\right)^{-1}$, and $\left(-a_{22}-d_{2} \Delta\right)^{-1}$ are the inverses of the operators $\left(a_{11}-d_{1} \Delta\right)$ and $\left(-a_{22}-d_{2} \Delta\right)$ in $\mathbf{Y}$ with the homogeneous Neumann boundary conditions. Then the system (3.1) is equivalent to the equation $(\mathbf{I}-\boldsymbol{F}) \mathbf{e}=0$. To apply the index theory, we investigate the eigenvalue of the problem

$$
-\left(\mathrm{I}-\mathcal{F}_{\mathrm{e}}\left(\mathrm{e}_{*}\right)\right) \Psi=\tilde{\mu} \Psi, \quad \boldsymbol{\Psi} \neq \mathbf{0},
$$

where $\Psi=\left(\psi_{1}, \psi_{2}\right)^{T}$ and $\mathbf{e}_{*}=\left(u_{*}, v_{*}\right)^{T}$. If 0 is not an eigenvalue of (3.16), then the LeraySchauder Theorem [27] implies that

$$
\text { index }\left(\mathbf{I}-\boldsymbol{F}, \mathbf{e}_{*}\right)=(-1)^{\gamma} \text {, }
$$

where $\gamma$ is the sum of the algebraic multiplicities of the positive eigenvalues of $-\left(\mathbf{I}-\mathscr{F}_{\mathbf{e}}\left(\mathbf{e}_{*}\right)\right)$, (3.16) can be rewritten as

$$
\begin{aligned}
& -(\tilde{\mu}+1) d_{1} \Delta \psi_{1}=(-\tilde{\mu}+1) a_{11} \psi_{1}+a_{12} \psi_{2} \\
& -(\tilde{\mu}+1) d_{2} \Delta \psi_{2}=a_{21} \psi_{1}+(\tilde{\mu}+1) a_{22} \psi_{2} .
\end{aligned}
$$

As in the proof of Theorem 2.5, we can conclude that $\tilde{\mu}$ is an eigenvalue of $-\left(\mathbf{I}-\mathcal{F}_{\mathbf{e}}\left(\mathbf{e}_{*}\right)\right)$ on $\mathbf{X}_{i j}$ if and only if it is a root of the characteristic equation $\operatorname{det} B_{i}=0$, where

$$
B_{i}=\left(\begin{array}{cc}
(-\tilde{\mu}+1) a_{11}-(\tilde{\mu}+1) d_{1} \mu_{i} & a_{12} \\
a_{21} & (\tilde{\mu}+1) a_{22}-(\tilde{\mu}+1) d_{2} \mu_{i}
\end{array}\right) .
$$

The characteristic equation $\operatorname{det} B_{i}=0$ can be written as

$$
\tilde{\mu}^{2}+\frac{2 d_{1} \mu_{i}}{a_{11}+d_{1} \mu_{i}} \tilde{\mu}+\frac{-d_{2} \mu_{i}\left(a_{11}-d_{1} \mu_{i}\right)+d_{1} \mu_{i}+\operatorname{det} J_{2}}{\left(a_{11}+d_{1} \mu_{i}\right)\left(-a_{22}+d_{2} \mu_{i}\right)}=0
$$

Note that $-d_{2} \mu_{i}\left(a_{11}-d_{1} \mu_{i}\right)+d_{1} \mu_{i}+\operatorname{det} J_{2}=Q_{i}$, where $Q_{i}$ is given in (2.23). Therefore, if 0 is not a root of (3.20) for all $i \geq 1$, we have

$$
\text { index }\left(\mathbf{I}-\boldsymbol{F}, \mathbf{e}_{*}\right)=(-1)^{r} \text {, }
$$

where $\gamma$ is the sum of the algebraic multiplicities of the positive roots of (3.20).

Theorem 3.5. Assume that the parameters $\lambda, \beta, m, n$, and $d_{1}$ are fixed and $0<a_{11}<1$. If $a_{11} / d_{1} \in$ $\left(\mu_{n}, \mu_{n+1}\right)$ for some $n \geq 2$ and $\sum_{2 \leq i \leq n, Q_{i}<0} \operatorname{dim} E\left(\mu_{i}\right)$ is odd, then the problem (3.1) has at least one non-constant positive solution for any $d_{2}>\tilde{d}_{2}$, where $Q_{i}$ and $\tilde{d}_{2}$ are given in (2.23) and (2.17), respectively. 
Proof. The proof, which is by contradiction, is based on the homotopy invariance of the topological degree. Suppose, on the contrary, that the assertion is not true for some $d_{2}=$ $\breve{d}_{2}>\tilde{d}_{2}$. In the follow we fix $d_{2}=\breve{d}_{2}$. Taking $d=a_{11} / \mu_{2}$ in Theorems 3.3 and 3.4, we obtain a positive constant $D$. Fixed $\widehat{d}_{1}=D+1$ and $\widehat{d}_{2}=1$. For $\theta \in[0,1]$, define a homotopy

$$
\boldsymbol{F}(\theta ; \mathbf{e}) \triangleq\left(\begin{array}{c}
\left(a_{11}-\left(\theta d_{1}+(1-\theta) \widehat{d}_{1}\right) \Delta\right)^{-1}\left(g_{1}(u, v)+a_{11} u\right) \\
\left(-a_{22}-\left(\theta d_{2}+(1-\theta) \hat{d}_{2}\right) \Delta\right)^{-1}\left(g_{2}(u, v)-a_{22} v\right)
\end{array}\right)
$$

Then, $\mathbf{e}$ is a positive solution of (3.1) if and only if it is a positive solution of $\boldsymbol{F}(1 ; \mathbf{e})=\mathbf{e}$. It is obvious that $\mathbf{e}_{*}$ is the unique constant positive solution of (3.22) for any $0 \leq \theta \leq 1$. By Theorem 3.3, there exists a positive constant $C$ such that, for all $0 \leq \theta \leq 1$, the positive solutions of the problem $\mathcal{F}(\theta ; \mathbf{e})=\mathbf{e}$ are contained in $B(C) \triangleq\left\{\mathbf{e} \in \mathbf{Y} \mid C^{-1}<u, v<C\right\}$. Since $\mathcal{F}(\theta ; \mathbf{e}) \neq \mathbf{e}$ for all $\mathbf{e} \in \partial B(C)$ and $\mathcal{F}(\theta ; \cdot): B(C) \times[0,1] \rightarrow \mathbf{Y}$ is compact, we can see that the degree $\operatorname{deg}(\mathbf{I}-\mathcal{F}(\theta ; \cdot), B(C), 0)$ is well defined. Moreover, by the homotopy invariance property of the topological degree, we have

$$
\operatorname{deg}(\mathbf{I}-\mathcal{F}(0 ; \cdot), B(C), 0)=\operatorname{deg}(\mathbf{I}-\mathcal{F}(1 ; \cdot), B(C), 0) .
$$

If $a_{11} / d_{1} \in\left(\mu_{n}, \mu_{n+1}\right)$ for some $n \geq 2$, then $i_{0}=n$ and $\tilde{d}_{2}=\min _{2 \leq i \leq n} d_{2}^{(i)}$ in (2.17). Since $d_{2}=\breve{d}_{2}>\tilde{d}_{2}$, then $Q_{k}<0$ for some $k, 2 \leq k \leq n$. Let $i=k$. Then, (3.20) has one positive root and a negative root. Furthermore, we have $Q_{i}>0$ for $i=1$ and all $i \geq n+1$. Therefore, when $i=1$ and $i \geq n+1$, the characteristic equation (3.20) has no roots with non-negative real parts. In addition, if $\sum_{2 \leq i \leq n, Q_{i}<0} \operatorname{dim} E\left(\mu_{i}\right)$ is odd, we have

$$
\text { index }\left(\mathbf{I}-\mathcal{F}(1 ; \cdot), \mathbf{e}_{*}\right)=(-1)^{\sum_{2<i \leq n, Q_{i}<0} \operatorname{dim} E\left(\mu_{i}\right)}=-1 \text {. }
$$

By our supposition, the equation $\mathcal{F}(1 ; \mathbf{e})=\mathbf{e}$ has only the positive solution $\mathbf{e}_{*}$ in $B(C)$, and hence

$$
\operatorname{deg}(\mathbf{I}-\mathcal{F}(1 ; \cdot), B(C), 0)=\operatorname{index}\left(\mathbf{I}-\mathcal{F}(1 ; \cdot), \mathbf{e}_{*}\right)=-1
$$

Similar argument shows $\tilde{\mu}$ is an eigenvalue of $-\left(\mathbf{I}-\mathcal{F}_{\mathbf{e}}\left(0 ; \mathbf{e}_{*}\right)\right)$ if and only if it is a root of the characteristic equation

$$
\tilde{\mu}^{2}+\frac{2 \widehat{d}_{1} \mu_{i}}{a_{11}+\widehat{d}_{1} \mu_{i}} \tilde{\mu}+\frac{-\widehat{d}_{2} \mu_{i}\left(a_{11}-\widehat{d}_{1} \mu_{i}\right)+\widehat{d}_{1} \mu_{i}+\operatorname{det} J_{2}}{\left(a_{11}+\widehat{d}_{1} \mu_{i}\right)\left(-a_{22}+\widehat{d}_{2} \mu_{i}\right)}=0 .
$$

It is easy to check that all eigenvalues of (3.26) have negative real parts for all $i \geq 1$, which implies

$$
\text { index }\left(\mathbf{I}-\mathcal{F}(0 ; \cdot), \mathbf{e}_{*}\right)=(-1)^{0}=1 \text {. }
$$


In view of Theorem 3.4, it follows that the equation $\mathcal{F}(0 ; \mathbf{e})=\mathbf{e}$ has only the positive solution $\mathbf{e}_{*}$ in $B(C)$, and therefore,

$$
\operatorname{deg}(\mathbf{I}-\mathcal{F}(0 ; \cdot), B(C), 0)=\operatorname{index}\left(\mathbf{I}-\mathcal{F}(0 ; \cdot), \mathbf{e}_{*}\right)=1
$$

This contradicts (3.23), and the proof is complete.

Example 3.6. Let $\Omega=(0,1)$. Then, the parameters $\lambda=2, \beta=6, m=3, n=0.1, d_{1}=0.0152$, and $d_{2}=4.1309$ satisfy all the conditions of Theorem 3.5. This means that $\left(u_{*}, v_{*}\right)=((2 \sqrt{159}-$ $4) / 31,(2 \sqrt{ } 159-4) / 31)$ is a locally asymptotically stable equilibrium point for the system

$$
\begin{gathered}
\frac{d u}{d t}=2 u-u^{2}-\frac{6 u v}{1+3 u+0.1 v} \\
\frac{d v}{d t}=v\left(1-\frac{v}{u}\right)
\end{gathered}
$$

but it is an unstable steady state for the system

$$
\begin{gathered}
u_{t}-0.0152 u_{x x}=2 u-u^{2}-\frac{6 u v}{1+3 u+0.1 v}, \quad x \in(0,1), t>0, \\
v_{t}-4.1309 v_{x x}=v\left(1-\frac{v}{u}\right), \quad x \in(0,1), t>0, \\
u_{x}=v_{x}=0, \quad x=0,1, t>0, \\
u(x, 0)=u_{0}(x)>0, \quad v(x, 0)=v_{0}(x) \geq 0, \quad x \in(0,1) .
\end{gathered}
$$

Moreover, the above reaction-diffusion system has at least one non-constant positive steady state.

\subsection{Bifurcation}

In this subsection, we discuss the bifurcation of non-constant positive solutions of (3.1) with respect to the diffusion coefficient $d_{2}$. In the consideration of bifurcation with respect to $d_{2}$, we recall that, for a constant solution $\mathbf{e}_{*},\left(\bar{d}_{2} ; \mathbf{e}_{*}\right) \in(0,+\infty) \times \mathbf{Y}$ is a bifurcation point of (3.1) if, for any $\delta \in\left(0, \bar{d}_{2}\right)$, there exists a $d_{2} \in\left[\bar{d}_{2}-\delta, \bar{d}_{2}+\delta\right]$ such that (3.1) has a non-constant positive solution close to $\mathbf{e}_{*}$. Otherwise, we say that $\left(\bar{d}_{2} ; \mathbf{e}_{*}\right)$ is a regular point [27].

We will consider the bifurcation of (3.1) at the equilibrium points $\left(\bar{d}_{2} ; \mathbf{e}_{*}\right)$, while all other parameters are fixed. From (2.23), we define

$$
Q\left(d_{2} ; \mu\right)=d_{1} d_{2} \mu^{2}-\left(d_{2} a_{11}-d_{1}\right) \mu+\operatorname{det} J_{2} .
$$

It is clear that $Q\left(d_{2} ; \mu\right)=0$ has at most two roots for any fixed $d_{2}>0$. Noting that $\operatorname{det} J_{2}>0$ in the proof of Theorem 2.4, if

$$
R\left(d_{2}\right) \triangleq\left(d_{2} a_{11}+d_{1}\right)^{2}+4 d_{1} d_{2} a_{12}>0,
$$


then $Q\left(d_{2}, \mu\right)=0$ has two different real roots with same symbols. Let

$$
\begin{gathered}
S_{p}=\left\{\mu_{1}, \mu_{2}, \mu_{3}, \ldots\right\}, \quad \Sigma\left(d_{2}\right)=\left\{\mu_{i}>0 \mid Q\left(d_{2} ; \mu_{i}\right)=0, d_{1} \mu_{i}<a_{11}\right\}, \\
\Gamma=\left\{d_{2} \mid d_{2}=d_{2}^{(i)}=\frac{d_{1} \mu_{i}-\operatorname{det} J_{2}}{\mu_{i}\left(a_{11}-d_{1} \mu_{i}\right)}, \mu_{i}>0, d_{1} \mu_{i}<a_{11}\right\} .
\end{gathered}
$$

We note that for each $d_{2}>0, \Sigma\left(d_{2}\right)$ may have 0 or 2 elements. The result is contained in the following theorem. Its proof is based on the topological degree arguments used earlier in this paper. We shall omit it but refer the reader to similar treatments in [24, 32, 33].

Theorem 3.7 (bifurcation with respect to $d_{2}$ ).

(1) Suppose that $\bar{d}_{2} \notin \Gamma$. Then, $\left(\bar{d}_{2} ; \mathbf{e}_{*}\right)$ is a regular point of (3.1).

(2) Suppose that $\bar{d}_{2} \in \Gamma$ and $R\left(\bar{d}_{2}\right)>0$. If $\sum_{\mu_{i} \in \Sigma\left(\bar{d}_{2}\right)} \operatorname{dim} E\left(\mu_{i}\right)$ is odd, then $\left(\bar{d}_{2} ; \mathbf{e}_{*}\right)$ is a bifurcation point of (3.1) with respect to the curve $\left(d_{2} ; \mathbf{e}_{*}\right), d_{2}>0$. In this case, there exists an interval $\left(\sigma_{1}, \sigma_{2}\right) \subset \mathbf{R}^{+}$, where

(i) $\bar{d}_{2}=\sigma_{1}<\sigma_{2}<+\infty$ and $\sigma_{2} \in \Gamma$ or

(ii) $0<\sigma_{1}<\sigma_{2}=\bar{d}_{2}$ and $\sigma_{1} \in \Gamma$ or

(iii) $\left(\sigma_{1}, \sigma_{2}\right)=\left(\bar{d}_{2},+\infty\right)$, or

(iv) $\left(\sigma_{1}, \sigma_{2}\right)=\left(0, \bar{d}_{2}\right)$,

such that for every $d_{2} \in\left(\sigma_{1}, \sigma_{2}\right)$, (3.1) admits a non-constant positive solution.

\section{Stationary Patterns for the PDE System with Cross-Diffusion}

In this section, we discuss the corresponding steady-state problem of the system (1.5):

$$
\begin{gathered}
-d_{1} \Delta u=\lambda u-u^{2}-\frac{\beta u v}{1+m u+n v} \quad \text { in } \Omega, \\
-d_{2} \Delta\left(1+d_{3} u\right) v=v\left(1-\frac{v}{u}\right) \quad \text { in } \Omega, \\
\partial_{v} u=\partial_{v} v=0 \quad \text { on } \partial \Omega .
\end{gathered}
$$

The existence and non-existence of the non-constant positive solutions of (4.1) will be given.

\subsection{A Priori Upper and Lower Bounds}

Theorem 4.1. If $d_{1}, d_{2} \geq d$ and $d_{3} / d_{2} \leq D$, where $d$ and $D$ are fixed positive numbers. Then, there exist positive constants $\underline{C}(\Lambda, d, D), \bar{C}(\Lambda, d, D)$ such that every positive solution $(u, v)$ of $(4.1)$ satisfies

$$
\underline{C}<u(x), v(x)<\bar{C}(\Lambda, d, D), \quad \forall x \in \bar{\Omega} .
$$


Proof. We first prove that there exists a positive constant $C=C(\Lambda, d, D)$ such that

$$
\max _{\bar{\Omega}} u \leq C \min _{\bar{\Omega}} u, \quad \max _{\bar{\Omega}} v \leq C \min _{\bar{\Omega}} v .
$$

A direct application of Lemma 3.1 to the first equation of (4.1) gives $u<\lambda$ on $\bar{\Omega}$. From Lemma 3.2, we have $\max _{\bar{\Omega}} u \leq C \min _{\bar{\Omega}} u$ for some positive constant $C(\Lambda, d, D)$. Define $\varphi(x)=d_{2}\left(1+d_{3} u\right) v$ and $\varphi\left(x_{0}\right)=\max _{\bar{\Omega}} \varphi$. Applying Lemma 3.1 again to the second equation of (4.1), we have $v\left(x_{0}\right) \leq u\left(x_{0}\right)<\lambda$, which implies

$$
\max _{\bar{\Omega}} v \leq d_{2}^{-1} \max _{\bar{\Omega}} \varphi<\left(1+d_{3} \lambda\right) \lambda
$$

On the other hand, $\varphi$ satisfies

$$
\begin{gathered}
-\Delta \varphi=\frac{u-v}{d_{2}\left(1+d_{3} u\right) u} \varphi \quad \text { in } \Omega, \\
\frac{\partial \varphi}{\partial v}=0 \quad \text { on } \partial \bar{\Omega} .
\end{gathered}
$$

Denote $c(x)=(u-v) /\left(d_{2}\left(1+d_{3} u\right) u\right)$. we have

$$
\begin{aligned}
\|c(x)\|_{\infty} & \leq \frac{1}{d_{2}}+\frac{\max _{\bar{\Omega}} v}{d_{2} \min _{\bar{\Omega}} u} \leq \frac{1}{d_{2}}+\frac{\left(1+d_{3} u\left(x_{0}\right)\right) v\left(x_{0}\right)}{d_{2} \min _{\bar{\Omega}} u} \\
& <\frac{1}{d_{2}}+\frac{\left(1+d_{3} \lambda\right) u\left(x_{0}\right)}{d_{2} \min _{\bar{\Omega}} u} \leq \frac{1}{d_{2}}+\frac{\left(1+d_{3} \lambda\right)}{d_{2}} \cdot \frac{\max _{\bar{\Omega}} u}{\min _{\bar{\Omega}} u} \leq C(\Lambda, d, D) .
\end{aligned}
$$

Hence, Lemma 3.2 implies that there exists a positive constant $C^{\prime}(\Lambda, d, D)$ such that $\max _{\bar{\Omega}} \varphi \leq$ $C^{\prime} \min _{\bar{\Omega}} \varphi$. Moreover, we have

$$
\frac{\max _{\bar{\Omega}} v}{\min _{\bar{\Omega}} v} \leq \frac{\max _{\bar{\Omega}} \varphi}{\min _{\bar{\Omega}} \varphi} \cdot \frac{\max _{\bar{\Omega}}\left(1+d_{3} u\right)}{\min _{\bar{\Omega}}\left(1+d_{3} u\right)} \leq C^{\prime} \cdot \frac{\max _{\bar{\Omega}} u}{\min _{\bar{\Omega}} u} \leq C .
$$

Thus, (4.3) is proved.

Note that $\min _{\bar{\Omega}} v<v\left(x_{0}\right) \leq u\left(x_{0}\right) \leq \max _{\bar{\Omega}} u<\lambda$, (4.3) implies that there exists a positive constant $\bar{C}(\Lambda, d, D)$ such that $u(x), v(x)<\bar{C}$, for all $x \in \bar{\Omega}$.

Turn now to the lower bound. Suppose, on the contrary, that the first result of (4.1) does not hold. Then, there exists a sequence $\left\{d_{1, i}, d_{2, i}, d_{3, i}\right\}_{i=1}^{\infty}$ with $d_{1, i}, d_{2, i} \in[d,+\infty) \times[d,+\infty)$, $d_{3, i} \in(0,+\infty)$ such that the corresponding positive solutions $\left(u_{i}, v_{i}\right)$ of $(4.1)$ satisfy

$$
\min _{\bar{\Omega}} u_{i} \longrightarrow 0 \quad \text { or } \quad \min _{\bar{\Omega}} v_{i} \longrightarrow 0, \quad \text { as } i \longrightarrow \infty \text {, }
$$


and $\left(u_{i}, v_{i}\right)$ satisfies

$$
\begin{gathered}
-d_{1, i} \Delta u_{i}=\lambda u_{i}-u_{i}^{2}-\frac{\beta u_{i} v_{i}}{1+m u_{i}+n v_{i}} \quad \text { in } \Omega, \\
-d_{2, i} \Delta\left(1+d_{3, i} u_{i}\right) v_{i}=v_{i}\left(1-\frac{v_{i}}{u_{i}}\right) \quad \text { in } \Omega, \\
\partial_{\nu} u_{i}=\partial_{\nu} v_{i}=0 \quad \text { on } \partial \Omega .
\end{gathered}
$$

Integrating by parts, we obtain that

$$
\begin{gathered}
\int_{\Omega} u_{i}\left(\lambda-u_{i}-\frac{\beta v_{i}}{1+m u_{i}+n v_{i}}\right) d x=0, \\
\int_{\Omega} v_{i}\left(1-\frac{v_{i}}{u_{i}}\right) d x=0 .
\end{gathered}
$$

By the second equation of (4.10), there exists $x_{i} \in \Omega$ such that $v_{i}\left(x_{i}\right)=u_{i}\left(x_{i}\right)$, for all $i \geq 1$. By (4.8), this implies that

$$
\min _{\bar{\Omega}} u_{i} \longrightarrow 0, \quad \min _{\bar{\Omega}} v_{i} \longrightarrow 0 \quad \text { as } i \longrightarrow \infty
$$

Combining (4.3) yields

$$
\max _{\bar{\Omega}} u_{i} \longrightarrow 0, \quad \max _{\bar{\Omega}} v_{i} \longrightarrow 0 \quad \text { as } i \longrightarrow \infty
$$

So we have

$$
\lambda-u_{i}-\frac{\beta v_{i}}{1+m u_{i}+n v_{i}}>0 \quad \text { on } \bar{\Omega}, \forall i \gg 1
$$

Integrating the first equation of (4.9) over $\Omega$ by parts, we have

$$
\int_{\Omega} u_{i}\left(\lambda-u_{i}-\frac{\beta v_{i}}{1+m u_{i}+n v_{i}}\right) d x>0, \quad \forall i \gg 1
$$

which is a contradiction to the first equation of (4.10). The proof is completed.

\subsection{Non-Existence of Non-Constant Positive Steady States}

Theorem 4.2. If $d_{2}>1 / \mu_{2}$ and $d_{3} / d_{2} \leq D$, where $D$ is a fixed positive number, then the problem (4.1) has no non-constant positive solution if $d_{1}$ is sufficiently large. 
Proof. Assume that $(u, v)$ is a positive solution of (4.1), multiplying the two equations of (4.1) by $(u-\bar{u})$ and $(v-\bar{v})$ respectively, and then integrating over $\Omega$ by parts, we have

$$
\begin{aligned}
& \int_{\Omega}\left\{d_{1}|\nabla u|^{2}+d_{2}\left(1+d_{3} u\right)|\nabla v|^{2}+d_{2} d_{3} v \nabla u \cdot \nabla v\right\} d x \\
& =\int_{\Omega}\left\{\lambda-(u+\bar{u})-\frac{\beta(n \bar{v}+1)}{(1+m \bar{u}+n \bar{v})(1+m u+n v)}\right\}(u-\bar{u})^{2} d x \\
& \quad+\int_{\Omega}\left\{-\frac{\beta(m u+1) \bar{u}}{(1+m \bar{u}+n \bar{v})(1+m u+n v)}+\frac{\bar{v}^{2}}{u \bar{u}}\right\}(u-\bar{u})(v-\bar{v}) d x+\int_{\Omega}\left(1-\frac{v+\bar{v}}{u}\right)(v-\bar{v})^{2} d x .
\end{aligned}
$$

From Theorem 4.1 and Young's inequality, we obtain

$$
\begin{aligned}
& \int_{\Omega}\left\{d_{1}|\nabla u|^{2}+d_{2}\left(1+d_{3} u\right)|\nabla v|^{2}\right\} d x \\
& \quad \leq \int_{\Omega}\left\{C(\varepsilon)(u-\bar{u})^{2}+(1+\varepsilon)(v-\bar{v})^{2}+\frac{d_{2}^{2} d_{3}^{2} v^{2}}{4 \varepsilon}|\nabla u|^{2}+\varepsilon|\nabla v|^{2}\right\} d x
\end{aligned}
$$

for some positive constant $C(\varepsilon)$ only depending on $\Lambda, \varepsilon, D$. By this combined with Theorem 4.1 and Poincaré inequality, we obtain

$$
\int_{\Omega}\left\{d_{1}|\nabla u|^{2}+d_{2}\left(1+d_{3} u\right)|\nabla v|^{2}\right\} d x \leq \int_{\Omega}\left\{C(\varepsilon)\left(1+d_{2}^{2} d_{3}^{2}\right)|\nabla u|^{2}+\left(\frac{1}{\mu_{2}}+\varepsilon\right)|\nabla v|^{2}\right\} d x
$$

which implies that $(u, v)=(\bar{u}, \bar{v})$ if $d_{1}>C\left(1+d_{2}^{2} d_{3}^{2}\right), d_{2}>\left(1 / \mu_{2}\right)+\varepsilon$ and $d_{3} / d_{2} \leq D$.

\subsection{Existence of Non-Constant Positive Steady States}

To show the existence of non-constant positive solutions, we use Leray-Schauder degree theory again. Denote $w=\left(1+d_{3} u\right) v$ and $w_{*}=\left(1+d_{3} \mathcal{u}_{*}\right) v_{*}$, then (4.1) can be rewritten as

$$
\begin{gathered}
-d_{1} \Delta u=\lambda u-u^{2}-\frac{\beta u w}{\left(1+d_{3} u\right)(1+m u)+n w} \triangleq \bar{g}_{1}(u, w) \quad \text { in } \Omega, \\
-d_{2} \Delta w=\frac{w}{1+d_{3} u}\left(1-\frac{w}{\left(1+d_{3} u\right) u}\right) \triangleq \bar{g}_{2}(u, w) \quad \text { in } \Omega \\
\partial_{v} u=\partial_{v} v=0 \quad \text { on } \partial \Omega .
\end{gathered}
$$


So, (4.18) has a unique positive constant solution $h_{*} \triangleq\left(u_{*}, w_{*}\right)$. The linearization matrix of $\overline{\mathrm{G}}(u, w)=\left(\bar{g}_{1}(u, w), \bar{g}_{2}(u, w)\right)^{T}$ at $\left(u_{*}, v_{*}\right)$ is

$$
J_{3}=\left(\begin{array}{ll}
m_{11} & m_{12} \\
m_{21} & m_{22}
\end{array}\right)
$$

where

$$
\begin{aligned}
m_{11}=a_{11}-a_{12} \frac{d_{3} u_{*}}{1+d_{3} u_{*}}, & m_{12}=\frac{a_{12}}{1+d_{3} u_{*}}, \\
m_{21}=1+\frac{d_{3} u_{*}}{1+d_{3} u_{*}}, & m_{22}=-\frac{1}{1+d_{3} u_{*}}
\end{aligned}
$$

If

$$
m_{11}=a_{11}-a_{12} \frac{d_{3} u_{*}}{1+d_{3} u_{*}}>0
$$

we can define a compact operator $\Phi: \mathrm{Y} \rightarrow \mathrm{Y}$ by

$$
\Phi(\mathbf{h}) \triangleq\left(\begin{array}{c}
\left(m_{11}-d_{1} \Delta\right)^{-1}\left(\bar{g}_{1}(u, w)+m_{11} u\right) \\
\left(-m_{22}-d_{2} \Delta\right)^{-1}\left(\bar{g}_{2}(u, w)-m_{22} w\right)
\end{array}\right)
$$

where $\mathbf{h}=(u(x), w(x))^{T},\left(m_{11}-d_{1} \Delta\right)^{-1}$, and $\left(-m_{22}-d_{2} \Delta\right)^{-1}$ are the inverses of the operators $\left(m_{11}-d_{1} \Delta\right)$ and $\left(-m_{22}-d_{2} \Delta\right)$ in $\mathbf{Y}$ with the homogeneous Neumann boundary condition. Moreover, the system (4.18) is equivalent to the equation $(\mathbf{I}-\Phi) \mathbf{h}=0$. To apply the index theory, we investigate the eigenvalue of the problem

$$
-\left(\mathbf{I}-\Phi_{\mathbf{h}}\left(\mathbf{h}_{*}\right)\right) \Psi=\tilde{\mu} \Psi, \quad \Psi \neq \mathbf{0},
$$

where $\Psi=\left(\psi_{1}, \psi_{2}\right)^{T}$. If 0 is not an eigenvalue of (4.23), then the Leray-Schauder Theorem implies that

$$
\operatorname{index}\left(\mathbf{I}-\Phi, \mathbf{h}_{*}\right)=(-1)^{r},
$$

where $\gamma$ is the sum of the algebraic multiplicities of the positive eigenvalues of $-\left(\mathbf{I}-\Phi_{\mathbf{h}}\left(\mathbf{h}_{*}\right)\right)$. Notice that (4.23) can be rewritten as

$$
\begin{aligned}
& -(\tilde{\mu}+1) d_{1} \Delta \psi_{1}=(-\tilde{\mu}+1) m_{11} \psi_{1}+m_{12} \psi_{2}, \\
& -(\tilde{\mu}+1) d_{2} \Delta \psi_{2}=m_{21} \psi_{1}+(\tilde{\mu}+1) m_{22} \psi_{2} .
\end{aligned}
$$


As the proof of Theorem 2.5, we can conclude that $\tilde{\mu}$ is an eigenvalue of $-\left(\mathbf{I}-\Phi_{\mathbf{h}}\left(\mathbf{h}_{*}\right)\right)$ on $\mathbf{X}_{i j}$ if and only if it is a root of the characteristic equation $\operatorname{det} \bar{B}_{i}=0$, where

$$
\bar{B}_{i}=\left(\begin{array}{cc}
(-\tilde{\mu}+1) m_{11}-(\tilde{\mu}+1) d_{1} \mu_{i} & m_{12} \\
m_{21} & (\tilde{\mu}+1) m_{22}-(\tilde{\mu}+1) d_{2} \mu_{i}
\end{array}\right)
$$

The characteristic equation $\operatorname{det} \bar{B}_{i}=0$ can be written as

$$
P_{i}(\tilde{\mu}) \triangleq \tilde{\mu}^{2}+M_{1}\left(d_{3} ; \mu_{i}\right) \tilde{\mu}+M_{2}\left(d_{3} ; \mu_{i}\right)=0,
$$

where

$$
M_{1}\left(d_{3} ; \mu_{i}\right)=\frac{2 d_{1} \mu_{i}}{m_{11}+d_{1} \mu_{i}}, \quad M_{2}\left(d_{3} ; \mu_{i}\right)=\frac{d_{1} d_{2} \mu_{i}{ }^{2}-\left(d_{1} m_{22}+d_{2} m_{11}\right) \mu_{i}+\operatorname{det} J_{3}}{\left(m_{11}+d_{1} \mu_{i}\right)\left(-m_{22}+d_{2} \mu_{i}\right)} .
$$

When $i=1$,

$$
P_{1}(\tilde{\mu})=\tilde{\mu}^{2}-\frac{m_{11} m_{22}-m_{12} m_{21}}{m_{11} m_{22}}=\tilde{\mu}^{2}-\frac{a_{11}+a_{12}}{m_{11}} .
$$

In the following, we always assume that (4.21) holds. Note that $a_{11}+a_{a 12}=-\operatorname{det} J_{2}<0$, we can conclude that (4.29) has no root with positive real part.

When $i \geq 2, M_{1}\left(d_{3} ; \mu_{i}\right)>0$. Consider the following limit:

$$
\lim _{d_{3} \rightarrow+\infty} M_{2}\left(d_{3} ; \mu\right)=\frac{d_{1} \mu-\left(a_{11}-a_{12}\right)}{d_{1} \mu+a_{11}-a_{12}} .
$$

For sake of convenience, denote

$$
\widehat{\mu}=\frac{a_{11}-a_{12}}{d_{1}}, \quad \Lambda_{2}=\left\{(\lambda, \beta, m, n) \mid a_{11}<-a_{12}\right\} .
$$

Some meticulous computations and simple analysis indicate that the following lemma is true.

Lemma 4.3. Let $(\lambda, \beta, m, n) \in \Lambda_{2}$. Assume that $\hat{\mu} \in\left(\mu_{n}, \mu_{n+1}\right)$ for some $n \geq 2$ and the $\operatorname{sum} \sum_{i=2}^{n} \operatorname{dim} E\left(\mu_{i}\right)$ is odd. Then, there exists a positive constant $\bar{D}$ such that for $d_{3}>\bar{D}$, index $(\Phi(\cdot), \widetilde{\mathbf{h}})=-1$.

Theorem 4.4. Under the same assumption of Lemma 4.3, there exists a positive constant $\bar{D}$ such that for $d_{3}>\bar{D}$, the problem (4.1) has at least one non-constant positive solution.

Proof. From Lemma 4.3, there exists a positive constant $\bar{D}$ such that, when $d_{3}>\bar{D}$, $\operatorname{index}(\mathbf{F}(\cdot), \widetilde{\mathbf{u}})=-1$. We shall prove that for any $d_{3}>\bar{D},(4.1)$ has at least one non-constant positive solution. The proof, which is by contradiction, is based on the homotopy invariance of the topological degree. Suppose, on the contrary, that the assertion is not true for some 
$d_{3}=\widehat{d}_{3}>\bar{D}$. Hereafter, we fix $d_{3}=\widehat{d}_{3}$ and $\widehat{d}_{2}=1 / \mu_{2}+1$. Let $\widehat{d}_{1}$ be so large that the conditions in Theorem 4.2 hold for $d_{3}=0$. For $\theta \in[0,1]$, define

$$
\Phi(\theta ; \mathbf{h}) \triangleq\left(\begin{array}{c}
\left(m_{11}-\left[\theta d_{1}+(1-\theta) \widehat{d}_{1}\right] \Delta\right)^{-1}\left(\lambda u-u^{2}-\frac{\beta u w}{\left(1+\theta d_{3} u\right)(1+m u)+n w}+m_{11} u\right) \\
\left(-m_{22}-\left[\theta d_{2}+(1-\theta) \widehat{d}_{2}\right] \Delta\right)^{-1}\left(\frac{w}{1+\theta d_{3} u}\left(1-\frac{w}{\left(1+\theta d_{3} u\right) u}\right)-m_{22} w\right)
\end{array}\right)
$$

It is obvious that $\widetilde{\mathbf{h}}$ is the unique constant positive solution of (4.32) for any $0 \leq \theta \leq 1$. By Theorem 4.1 and $w=\left(1+d_{3} u\right) v$, there exists a positive constant $C$ such that, for all $0 \leq \theta \leq 1$, the positive solutions of the problem $\Phi(\theta ; \mathbf{h})=0$ are contained in $B(C) \triangleq\left\{\mathbf{h} \in \mathbf{Y} \mid C^{-1}<\right.$ $u, w<C\}$. Since $\Phi(\theta ; \mathbf{h}) \neq 0$ for all $\mathbf{h} \in \partial B(C)$, we can see that the degree $\operatorname{deg}(\Phi(\theta ; \cdot), B(C), 0)$ is well defined. Moreover, by the homotopy invariance property of the topological degree, we have

$$
\operatorname{deg}(\Phi(0 ; \cdot), B(C), 0)=\operatorname{deg}(\Phi(1 ; \cdot), B(C), 0)
$$

By our supposition and Lemma 4.3 , the equation $\Phi(1 ; \mathbf{h})=0$ has only the positive solution $\widetilde{\mathbf{h}}$ in $B(C)$, and hence $\operatorname{deg}(\Phi(1 ; \cdot), B(C), 0)=\operatorname{index}(\Phi(1 ; \cdot), \tilde{\mathbf{h}})=-1$. Similar argument shows $\operatorname{deg}(\Phi(0 ; \cdot), B(C), 0)=\operatorname{index}(\Phi(0 ; \cdot), \widetilde{\mathbf{h}})=1$. This contradicts with $(4.33)$, and then the proof is completed.

Example 4.5. Let $\Omega=(0,1)$. Then, the parameters $\lambda=2, \beta=6, m=3, n=0.1, d_{1}=0.0743$, $d_{2}=2$, and $d_{3}=100$ satisfy all the conditions of Theorem 4.4 . In this case, $\left(u_{*}, v_{*}\right)=((2 \sqrt{159}-$ $4) / 31,(2 \sqrt{ } 159-4) / 31)$ is a locally asymptotically stable steady state for the system

$$
\begin{gathered}
u_{t}-0.0743 u_{x x}=2 u-u^{2}-\frac{6 u v}{1+3 u+0.1 v}, \quad x \in(0,1), t>0, \\
v_{t}-2 v_{x x}=v\left(1-\frac{v}{u}\right), \quad x \in(0,1), t>0, \\
u_{x}=v_{x}=0, \quad x=0,1, t>0, \\
u(x, 0)=u_{0}(x)>0, \quad v(x, 0)=v_{0}(x) \geq 0, \quad x \in(0,1) .
\end{gathered}
$$

However, it is an unstable steady state for the system

$$
\begin{gathered}
u_{t}-0.0743 u_{x x}=2 u-u^{2}-\frac{6 u v}{1+3 u+0.1 v}, \quad x \in(0,1), t>0, \\
v_{t}-2(v+100 u v)_{x x}=v\left(1-\frac{v}{u}\right), \quad x \in(0,1), t>0, \\
u_{x}=v_{x}=0, \quad x=0,1, t>0, \\
u(x, 0)=u_{0}(x)>0, v(x, 0)=v_{0}(x) \geq 0, \quad x \in(0,1) .
\end{gathered}
$$


Moreover, the above cross-diffusion system has at least one non-constant positive steady state.

\section{Conclusions}

In this paper, we have introduced a more realistic mathematical model for a diffusive preypredator system where the Beddington-DeAngelis functional response is used only in the prey equation and a Leslie-Gower term is contained by the predator equation. This system admits rich dynamics which include the attractor, persistence, stable or unstable equilibria, and Turing patterns. Letting $n=0$, our conclusions are essentially the same as for the systems with a Holling-Tanner response for the prey $[7,8]$. However, the presence of mutual interference by predators can stabilize the positive equilibrium. Moreover, after the crossdiffusion pressure is introduced, our model is a strongly coupled reaction-diffusion system, which is mathematically more complex than systems without cross-diffusion. We show that, even though the unique positive constant steady state is asymptotically stable for the dynamics with diffusion, non-constant positive steady solutions can also exist due to the emergence of cross-diffusion. Our results confirm that cross-diffusion can create stationary patterns.

\section{Acknowledgments}

This paper was supported by the National Natural Science Foundation of China (no. 11061031, 10871160), the NSF of Gansu Province (no. 096RJZA118) and NWNU-KJCXGC03-47, 61 Foundations.

\section{References}

[1] H. Malchow, S. Petrovskii, and E. Venturino, Spatiotemporal Patterns in Ecology and Epidemiology, Chapman and Hall/CRC Press, Boca Raton, Fla, USA, 2008.

[2] R. S. Cantrell and C. Cosner, Spatial Ecology via Reaction-Diffusion Equations, John Wiley \& Sons, Chichester, UK, 2003.

[3] P. A. Braza, "The bifurcation structure of the Holling-Tanner model for predator-prey interactions using two-timing," SIAM Journal on Applied Mathematics, vol. 63, no. 3, pp. 889-904, 2003.

[4] S. Hsu and T. W. Huang, "Global stability for a class of predator-prey systems," SIAM Journal on Applied Mathematics, vol. 55, no. 3, pp. 763-783, 1995.

[5] Y. Du and S. B. Hsu, "A diffusive predator-prey model in heterogeneous environment," Journal of Differential Equations, vol. 203, no. 2, pp. 331-364, 2004.

[6] Y. Du and M. Wang, "Asymptotic behavior of positive steady states to a predator-prey model," Proceedings of the Royal Society of Edinburgh. Section A, vol. 136, no. 4, pp. 759-778, 2006.

[7] R. Peng and M. Wang, "Positive steady states of the Holling-Tanner prey-predator model with diffusion," Proceedings of the Royal Society of Edinburgh A, vol. 135, no. 1, pp. 149-164, 2005.

[8] R. Peng and M. Wang, "Global stability of the equilibrium of a diffusive Holling-Tanner prey-predator model," Applied Mathematics Letters, vol. 20, no. 6, pp. 664-670, 2007.

[9] W. Ko and K. Ryu, "Non-constant positive steady-states of a diffusive predator-prey system in homogeneous environment," Journal of Mathematical Analysis and Applications, vol. 327, no. 1, pp. 539 $549,2007$.

[10] R. Peng and M. Wang, "Qualitative analysis on a diffusive prey-predator model with ratio-dependent functional response," Science in China, Series A, vol. 51, no. 11, pp. 2043-2058, 2008.

[11] M. L. Rosenzweig, "Paradox of enrichment: destabilization of exploitation ecosystems in ecological time," Science, vol. 171, no. 3969, pp. 385-387, 1971. 
[12] J. Poggiale, M. Gauduchon, and P. Auger, "Enrichment paradox induced by spatial heterogeneity in a phytoplankton-Zooplankton system," Mathematical Modelling of Natural Phenomena, vol. 3, no. 3, pp. 87-102, 2008.

[13] P. A. Abrams and L. R. Ginzburg, "The nature of predation: prey dependent, ratio dependent or neither?" Trends in Ecology and Evolution, vol. 15, no. 8, pp. 337-341, 2000.

[14] J. Beddington, "Mutual interference between parasites or predators and its effect on searching efficiency," Journal of Animal Ecology, vol. 44, pp. 331-340, 1975.

[15] D. DeAngelis, R. A. Goldstein, and R. V. OıNeill, “A model for tropic interaction," Ecology, vol. 56, pp. 881-892, 1975.

[16] H. Leslie and J. Gower, "The properties of a stochastic model for the predator-prey type of interaction between two species," Biometrica, vol. 47, pp. 219-234, 1960.

[17] R. S. Cantrell and C. Cosner, "On the dynamics of predator-prey models with the BeddingtonDeAngelis functional response," Journal of Mathematical Analysis and Applications, vol. 257, no. 1, pp. 206-222, 2001.

[18] W. Chen and M. Wang, "Qualitative analysis of predator-prey models with Beddington-DeAngelis functional response and diffusion," Mathematical and Computer Modelling, vol. 42, no. 1-2, pp. 31-44, 2005.

[19] B. Dubey, B. Das, and J. Hussain, "A predator-prey interaction model with self and cross-diffusion," Ecological Modelling, vol. 141, no. 1-3, pp. 67-76, 2001.

[20] K. Kuto and Y. Yamada, "Multiple coexistence states for a prey-predator system with cross-diffusion," Journal of Differential Equations, vol. 197, no. 2, pp. 315-348, 2004.

[21] W. Chen and R. Peng, "Stationary patterns created by cross-diffusion for the competitor-competitormutualist model," Journal of Mathematical Analysis and Applications, vol. 291, no. 2, pp. 550-564, 2004.

[22] A. B. Ryabov and B. Blasius, "Population growth and persistence in a heterogeneous environment: the role of diffusion and advection," Mathematical Modelling of Natural Phenomena, vol. 3, no. 3, pp. 42-86, 2008.

[23] Y. Du, P. Y. H. Pang, and M. Wang, “Qualitative analysis of a prey-predator model with stage structure for the predator," SIAM Journal on Applied Mathematics, vol. 69, no. 2, pp. 596-620, 2008.

[24] P. Y. H. Pang and M. Wang, "Strategy and stationary pattern in a three-species predator-prey model," Journal of Differential Equations, vol. 200, no. 2, pp. 245-273, 2004.

[25] Y. Lou and W. M. Ni, "Diffusion, self-diffusion and cross-diffusion," Journal of Differential Equations, vol. 131, no. 1, pp. 79-131, 1996.

[26] A. Friedman, Partial Differential Equations of Parabolic Type, Prentice-Hall Press, New York, NY, USA, 1964.

[27] J. Smoller, Shock Waves and Reaction-Diffusion Equations, Springer, New York, NY, USA, 1983.

[28] W. M. Ni and M. Tang, "Turing patterns in the Lengyel-Epstein system for the CIMA reaction," Transactions of the American Mathematical Society, vol. 357, no. 10, pp. 3953-3969, 2005.

[29] P. Pang and M. Wang, "Qualitative analysis of a ratio-dependent predator-prey with diffusion," Proceedings of the Royal Society of Edinburgh. Section A, vol. 133, no. 4, pp. 919-942, 2003.

[30] Z. Lin and M. Pedersen, "Stability in a diffusive food-chain model with Michaelis-Menten functional response," Nonlinear Analysis, Theory, Methods and Applications, vol. 57, no. 3, pp. 421-433, 2004.

[31] C. S. Lin, W. M. Ni, and I. Takagi, "Large amplitude stationary solutions to a chemotaxis system," Journal of Differential Equations, vol. 72, no. 1, pp. 1-27, 1988.

[32] J. H. Wu, "Global bifurcation of coexistence state for the competition model in the chemostat," Nonlinear Analysis, Theory, Methods and Applications, vol. 39, no. 7, pp. 817-835, 2000.

[33] J. Jang, W. Ni, and M. Tang, "Global bifurcation and structure of Turing patterns in the 1D LengyelEpstein Model," Journal of Dynamics and Differential Equations, vol. 16, no. 2, pp. 297-320, 2004. 